\title{
Closure Operators for Order Structures ${ }^{\star}$
}

\author{
Ryszard Janicki ${ }^{1}$, Dai Tri Man Lế $\hat{e}^{\star \star 2}$, and Nadezhda Zubkova ${ }^{1}$ \\ 1 Department of Computing and Software, McMaster University, \\ Hamilton, Canada L8S 4K1 \\ \{janicki, zubkovna\}@mcmaster.ca \\ 2 Department of Computer Science, University of Toronto, \\ Toronto, Canada M5S 3G4 \\ ledt@cs.toronto.edu
}

\begin{abstract}
We argue that closure operators are fundamental tools for the study of relationships between order structures and their sequence representations. We also propose and analyse a closure operator for interval order structures.
\end{abstract}

\section{Introduction}

While the two major models of concurrency, interleaving abstraction $([2,22])$ and partially ordered causality $([5,15,23])$, have been very successful, they have some limitations. Neither of them can model the "not later than" relationship effectively, which causes problems with specifying priorities, error recovery, time testing, inhibitor nets, etc. (see for instance $[4,9,12,16-18]$ ). A solution, proposed independently (in this order) in $[19,8]$ and $[10]$, suggests modeling concurrent behaviours by a triple $(X, \prec, \sqsubset)$, where $X$ is the set of event occurrences, and $\prec$ and $\sqsubset$ are binary relations on $X$. The relation $\prec$ is "causality" (i.e. an abstraction of the "earlier than" relationship), and $\sqsubset$ is "weak causality" (an abstraction of the "not later than" relationship). For this model, the following two kinds of relational structures are of special importance: stratified order structures (so-structures) and interval order structures (io-structures). The former structures can fully model concurrent behaviours when system executions (operational semantics) are described in terms of stratified orders, while the latter structures can fully model concurrent behaviours when system executions are described in terms of interval orders [9,13]. It was argued in [11] (and also implicitly in 1914 Wiener's paper [26]) that any execution that can be observed by a single observer must be an interval order. Thus, io-structures provide a very general model of concurrency. However, the theory of io-structures is far less developed than the simpler theory of so-structures.

When dealing with partial orders, many constructions use the fundamental notion of transitive closure of relations. The analogue of transitive closure for so-structures, called $\diamond$-closure, has been proposed in [12] and successfully used in [12,16-18] and others. However, a similar concept for io-structures has not yet been proposed. In this paper we introduce the concept of -closure for io-structures and show that it has the same kind of properties as transitive closure and $\diamond$-closure.

\footnotetext{
* Partially supported by NSERC Grant of Canada.

${ }^{\star \star}$ Partially supported by Ontario Graduate Scholarship.
} 
The paper is structured as follows. Section 2 provides some mathematical preliminaries, while basic properties of Mazurkiewicz traces are discussed in Section 3. In Section 4 old and new properties of so-structures are discussed. Section 5 is devoted to io-structures and their $\$$-closure operator. Section 6 contains some final comments.

\section{Relations, Partial Orders and Transitive Closure}

In this section, we recall some well-known mathematical concepts and results that will be used frequently in this paper.

Let $X$ be a set and $R_{1}, R_{2} \subseteq X \times X$ are two relations on $X$. We define $R_{1} \circ R_{2} \stackrel{d f}{=}$ $\left\{(x, y) \mid \exists z \in Z .(x, z) \in R_{1} \wedge(y, z) \in R_{2}\right\}$, and $i d_{X} \stackrel{d f}{=}\{(x, x) \mid x \in X\}$. For each relation $R \subseteq X \times X$, we define $R^{+}$, the transitive closure of $R$, as $R^{+} \stackrel{d f}{=} \bigcup_{i=1}^{\infty} R^{i}$, and the reflexive and transitive closure of $R$, as $R^{*}=\bigcup_{i=0}^{\infty} R^{i}$, where $R^{0}=i d_{X}$ and $R^{i+1}=R^{i} \circ R$ for $i>0$.

A binary relation $R \subseteq X \times X$ is: irreflexive iff for all $a \in X . \neg(a R a)$; transitive iff for all $a, b, c \in X . a R b \wedge b R c \Longrightarrow a R c$; and acyclic iff for all $a \in X . \neg\left(a R^{+} a\right)$.

A relation $<\subseteq X \times X$ is a (strict) partial order if it is irreflexive and transitive, i.e. for all $a, c, b \in X, a \nless a$ and $a<b<c \Longrightarrow a<c$. We also define:

$$
\begin{aligned}
& a \frown<b \stackrel{d f}{\Longleftrightarrow} \neg(a<b) \wedge \neg(b<a) \wedge a \neq b \\
& a<\neg b \stackrel{d f}{\Longleftrightarrow} a<b \vee a \frown<b
\end{aligned}
$$

Note that $a \frown<b$ means $a$ and $b$ are incomparable (w.r.t. $<$ ) elements of $X$.

Let $<$ be a partial order on a set $x$. Then

1. $<$ is total if $\frown<=\emptyset$. In other words, for all $a, b \in X, a<b \vee b<a \vee a=b$. For clarity, we will reserve the symbol $\triangleleft$ to denote total orders;

2. < is stratified if $a \frown<b \frown<c \Longrightarrow a \frown<\vee \vee a=c$, i.e., the relation $\frown<\cup i d_{X}$ is an equivalence relation on $X$.

3. $<$ is interval if for all $a, b, c, d \in X, a<c \wedge b<d \Longrightarrow a<d \vee b<c$.

It is clear from these definitions that every total order is stratified and every stratified order is interval.

Given a partial order $<\subseteq X \times X$, a relation $<^{\prime} \subseteq X \times X$ is an extension of $<$ if $<\subseteq<^{\prime}$. For convenience, we define $\operatorname{Total}(<) \stackrel{d f}{=}\{\triangleleft \subseteq X \times X \mid \triangleleft$ is a total order and $<\subseteq \triangleleft\}$. In other words, the set Total $(<)$ consists of all the total order extensions of $<$.

By Szpilrajn's Theorem [25], we know that every partial order $<$ is uniquely represented by the the set Total $(<)$. Szpilrajn's Theorem can be stated as following:

Theorem 1 (Szpilrajn [25]). For every partial order $<,<=\bigcap_{\triangleleft \in \operatorname{Total}(<)} \triangleleft$.

Stratified orders are often defined in an alternative way, namely, a partial order $<$ on $X$ is stratified if and only if there exists a total order $\triangleleft$ on some $Y$ and a mapping $\phi: X \rightarrow Y$ such that $\forall x, y \in X . x<y \Longleftrightarrow \phi(x) \triangleleft \phi(y)$. This definition is illustrated in Figure 1, where $\phi(a)=\{a\}, \phi(b)=\phi(c)=\{b, c\}, \phi(d)=\{d\}$. Note that for all $x, y \in\{a, b, c, d\}$ we have $x<_{2} y \Longleftrightarrow \phi(x) \triangleleft_{2} \phi(y)$, where the total order $\triangleleft_{2}$ can be concisely represented by a step sequence $\{a\}\{b, c\}\{d\}$. As a consequence, stratified orders and step sequences can uniquely represent each other (cf. $[12,14,20]$ ).

For the interval orders, the name and intuition follow from Fishburn's Theorem: 

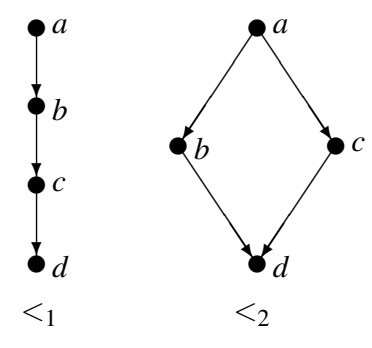

$<_{2}$

total

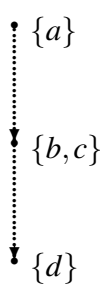

$\triangleleft_{2}$

total

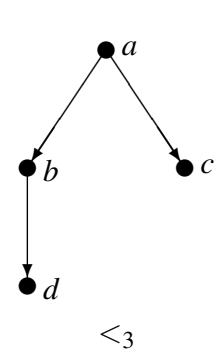

interval

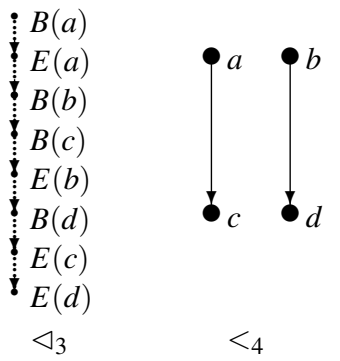

total not interval

Fig. 1. Various types of partial orders (represented as Hasse diagrams). The partial order $<_{1}$ is an extension of $<_{2},<_{2}$ is an extension of $<_{3}$, and $<_{3}$ is and extension of $<_{4}$. Note that order $<_{1}$, being total, is uniquely represented by a sequence $a b c d$, the stratified order $<_{2}$ is uniquely represented by a step sequence $\{a\}\{b, c\}\{d\}$, and the interval order $<_{3}$ is (not uniquely) represented by a sequence that represents $\triangleleft_{3}$, i.e. $B(a) E(a) B(b) B(c) E(b) B(d) E(c) E(d)$.

Theorem 2 (Fishburn [6]). A partial order $<$ on $X$ is interval iff there exists a total order $\triangleleft$ on some $T$ and two mappings $B, E: X \rightarrow T$ such that for all $x, y \in X$,
1. $B(x) \triangleleft E(y)$, and
2. $x<y \Longleftrightarrow E(x) \triangleleft B(y)$.

Usually $B(x)$ is interpreted as the beginning and $E(x)$ as the end of an interval $x$. The intuition of Fishburn's theorem is illustrated in Figure 1 with $<_{3}$ and $\triangleleft_{3}$. For all $x, y \in\{a, b, c, d\}$, we have $B(x) \triangleleft_{3} E(x)$ and $x<_{3} y \Longleftrightarrow E(x) \triangleleft_{3} B(y)$.

We will next recall the fundamental properties of transitive closure operator.

Proposition 1. Let $R \subseteq X \times X$.

1. If $R$ is irreflexive then $R \subseteq R^{+} \backslash i d_{X}$,

2. $\left(R^{+}\right)^{+}=R^{+}$,

3. $R^{+}$is a partial order if and only if $R^{+}$is irreflexive,

4. if $R$ is a partial order then $R^{+}=R$.

5. if $R$ is a partial order and $R_{0} \subseteq R$, then $R_{0}^{+}$is a partial order and $R_{0}^{+} \subseteq R$.

These properties were extended to the $\diamond$-closure operator for so-structures in [12] and will be extended to the -closure operator for io-structures in Section 5.

\section{Partial Orders Generated by Mazurkiewicz Traces}

A triple $(X, *, \mathbb{1})$, where $X$ is a set, $*$ is a total binary operation on $X$, and $\mathbb{1} \in X$, is called a monoid [3], if $(a * b) * c=a *(b * c)$ and $a * \mathbb{1}=\mathbb{1} * a=a$, for all $a, b, c \in X$.

A nonempty equivalence relation $\sim \subseteq X \times X$ is a congruence in the monoid $(X, *, \mathbb{1})$ if for all $a_{1}, a_{2}, b_{1}, b_{2} \in X, a_{1} \sim b_{1} \wedge a_{2} \sim b_{2} \Rightarrow\left(a_{1} * a_{2}\right) \sim\left(b_{1} * b_{2}\right)$.

The triple $(X / \sim, \circledast,[\mathbb{1}])$, where $[a] \circledast[b]=[a * b]$, is called the quotient monoid of $(X, *, 1)$ under the congruence $\sim$. The symbols $*$ and $\circledast$ are often omitted if this does not lead to any discrepancy. 
Let $M=(X, *, \mathbb{1})$ be a monoid and let $E Q=\left\{x_{i}=y_{i} \mid i=1, \ldots, n\right\}$ be a finite set of equations. Define $\equiv_{E Q}$ (or just $\equiv$ ) to be the least congruence on $M$ satisfying, $x_{i}=y_{i} \Longrightarrow x_{i} \equiv_{E Q} y_{i}$, for each equation $x_{i}=y_{i} \in E Q$. We call the relation $\equiv_{E Q}$ the congruence defined by $E Q$, or EQ-congruence.

The quotient monoid $M_{\equiv_{E Q}}=\left(X / \equiv_{E Q}, \circledast,[\mathbb{1}]\right)$, where $[x] \circledast[y]=[x * y]$, is called an equational monoid (see [14,20] for more details).

Monoids of Mazurkiewicz traces (or traces) (cf. [5, 21]) are equational monoids over sequences. The theory of traces has been utilised to tackle problems from quite diverse areas including combinatorics, graph theory, algebra, logic and, especially concurrency theory [5,21].

Applications of traces in concurrency theory are originated from the fact that traces are sequence representation of partial orders, which gives traces the ability to model "true concurrency" semantics. We will now recall the definition of a trace monoid.

Definition $1([\mathbf{5}, 21])$. Let $M=\left(E^{*}, *, \lambda\right)$ be a free monoid generated by $E$, and let the relation ind $\subseteq E \times E$ be an irreflexive and symmetric relation (called independency), and $E Q=\{a b=b a \mid(a, b) \in i n d\}$. Let $\equiv_{\text {ind }}$, called trace congruence, be the congruence defined by $E Q$. Then the equational monoid $M_{\equiv_{\text {ind }}}=\left(E^{*} / \equiv_{i n d}, \circledast,[\lambda]\right)$ is a monoid of traces. The pair $(E$,ind $)$ is called a trace alphabet.

We will omit the subscript ind from trace congruence if it causes no ambiguity.

Example 1. Let $E=\{a, b, c\}$, ind $=\{(b, c),(c, b)\}$, i.e., $E Q=\{b c=c b\}$. Given three sequences $s=a b c b c a, s_{1}=a b c$ and $s_{2}=b c a$, we can generate the traces $[s]=\{a b c b c a$, $a b c c b a, a c b b c a, a c b c b a, a b b c c a, a c c b b a\},\left[s_{1}\right]=\{a b c, a c b\}$ and $\left[s_{2}\right]=\{b c a, c b a\}$. Note that $[s]=\left[s_{1}\right] \circledast\left[s_{2}\right]$ since $[a b c b c a]=[a b c] \circledast[b c a]=[a b c * b c a]$.

Each trace represents a finite partial order in the following sense. For the trace $[s]$ from Example 1, we can define $\Sigma_{[s]}=\left\{a^{(1)}, b^{(1)}, c^{(1)}, b^{(2)}, c^{(2)}, a^{(2)}\right\}$ to be the set of all enumerated events occurring in $[s]$, where $a^{(1)}$ and $a^{(2)}$ simply denote the first and the second occurrences of $a$ respectively in the sequence $s_{1}$. Then the partially ordered set (poset) $\left(\Sigma_{[s]}, \prec_{[s]}\right)$ represented by $[s]$ is depicted in the diagram on the right (arcs inferred from transitivity are omitted for simplicity).

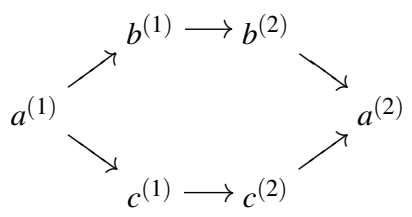

In fact, the total orders induced by the elements of $[s]$ comprise all the total extensions of $\prec_{[s]}$ (see [21]), which by Theorem 1 implies that $[s]$ uniquely determines the partial order $\prec_{[s]}$.

Remark 1. Given a sequence $s$, to construct the partial order $\prec_{[s]}$ represented by $[s]$, we do not need to build up to exponentially many elements of $[s]$. We can simply construct the direct acyclic graph $\left(\Sigma_{[s]}, \prec_{s}\right)$, where $x^{(i)} \prec_{s} y^{(j)}$ iff $x^{(i)}$ occurs before $y^{(j)}$ on the sequence $s$ and $(x, y) \notin$ ind. The relation $\prec_{s}$ is usually not the same as the partial order $\prec_{[s]}$. However, after applying the transitive closure operator, we have $\prec_{[s]}=\prec_{s}^{+}$. To extend this simple idea to the more difficult cases of constructing stratified or io-structures from their sequence representations, it is inevitable that we have to generalise the transitive closure operator to these order structures. 


\section{Stratified Order Structures, Comtraces, and $\diamond$-Closure}

A relational structure is a triple $S=\left(X, R_{1}, R_{2}\right)$, where $R_{1}, R_{2} \subseteq X \times X$. We will write $S=\left(X, R_{1}, R_{2}\right) \subseteq S^{\prime}=\left(X, R_{1}^{\prime}, R_{2}^{\prime}\right)$ iff $R_{1} \subseteq R_{1}^{\prime}$ and $R_{2} \subseteq R_{2}^{\prime}$.

Definition 2 ([11]). A stratified order structures (so-structure) is a relational structure $S=(X, \prec, \sqsubset)$, such that for all $a, b, c \in X$, the following hold:

$$
\begin{array}{llll}
\text { S1: } & a \not \subset a & \text { S3: } & a \sqsubset b \sqsubset c \wedge a \neq c \Longrightarrow a \sqsubset c \\
\text { S2: } & a \prec b \Longrightarrow a \sqsubset b & \text { S4: } & a \sqsubset b \prec c \vee a \prec b \sqsubset c \Longrightarrow a \prec c
\end{array}
$$

So-structures were independently introduced in [8] and [10]. Their comprehensive theory has been presented in $[12,13]$. They have been successfully applied to model inhibitor and priority systems, asynchronous races, synthesis problems, etc., [17] (see [9] for more references).

The relation $\prec$ is called causality and represents the "earlier than" relationship, and the relation $\sqsubset$ is called weak causality and represents the "not later than" relationship. The axioms S1-S4 model the mutual relationship between "earlier than" and "not later than" relations, provided that the system runs are defined as stratified orders.

A stratified order $<$ on $X$ is a stratified extension of a so-structure $S=(X, \prec, \sqsubset)$ if $\prec \subseteq<$ and $\sqsubset \subseteq<\urcorner$. The set of all stratified extensions of $S$ will be denoted by $\operatorname{Strat}(S)$.

Theorem 3 ([13]). For every so-structure $S=(X, \prec, \sqsubset)$ :

$$
S=\left(X, \bigcap_{<\in \operatorname{Strat}(S)}<, \bigcap_{<\in \operatorname{Strat}(S)}<\frown\right) .
$$

The above theorem is a generalisation of Szpilrajn's Theorem to so-structures and is interpreted as the proof of the claim that so-structures uniquely represent sets of equivalent system runs provided that the system operational semantics can be fully described in terms of stratified orders (see [9, 13] for details).

We will now present the concept of $\diamond$-closure that plays a substantial role in most of the applications of so-structures for modelling concurrent systems (cf. [13, 16, 17]).

Definition 3 ([12]). For every relational structure $S=\left(X, R_{1}, R_{2}\right)$ we define $S^{\diamond}$ as

$$
S^{\diamond} \stackrel{d f}{=}\left(X, \prec_{R_{1}, R_{2}}^{\diamond}, \sqsubset_{R_{1}, R_{2}}^{\diamond}\right)=\left(X,\left(R_{1} \cup R_{2}\right)^{*} \circ R_{1} \circ\left(R_{1} \cup R_{2}\right)^{*},\left(R_{1} \cup R_{2}\right)^{*} \backslash i d_{X}\right) .
$$

Intuitively the $\diamond$-closure is a generalisation of transitive closure for relations to sostructures. The theorem below shows that the $\diamond$-closure has all the properties formulated for transitive closure in Proposition 1.

Theorem 4 ([12]). Let $S=\left(X, R_{1}, R_{2}\right)$ be a relational structure.

1. If $R_{2}$ is irreflexive then $S \subseteq S^{\diamond}$.

2. $\left(S^{\diamond}\right)^{\diamond}=S^{\diamond}$.

3. $S \diamond$ is a so-structure if and only if the relation $\prec_{R_{1}, R_{2}}$ is irreflexive.

4. If $S$ is a so-structure then $S=S^{\diamond}$.

5. Let $S$ be a so-structure and let $S_{0} \subseteq S$. Then $S_{0}^{\diamond} \subseteq S$ and $S_{0}^{\diamond}$ is a so-structure. 
Among others, Theorem 4 helps us to show a relationship between so-structures and comtraces, an extension of Mazurkiewicz traces that allows us to model the "not later than" relationship using quotient monoids of step sequence monoids $[12,14,20]$.

Definition 4 ([12]). Let $E$ be a finite set (of events) and let $\operatorname{ser} \subseteq \operatorname{sim} \subset E \times E$ be two relations called serialisability and simultaneity respectively and the relation sim is irreflexive and symmetric. Then the triple $(E$, sim, ser $)$ is called the comtrace alphabet.

Intuitively, if $(a, b) \in \operatorname{sim}$ then $a$ and $b$ can occur simultaneously, while $(a, b) \in$ ser means that $a$ and $b$ may occur simultaneously or $a$ may occur before $b$ (i.e., both executions are equivalent). We define $\mathbb{S}$, the set of all (potential) steps, as the set of all cliques of the graph $(E$, sim $)$, i.e., $\mathbb{S} \stackrel{d f}{=}\{A \mid A \neq \emptyset \wedge \forall a, b \in A .(a=b \vee(a, b) \in \operatorname{sim})\}$. Hence, the triple $\left(\mathbb{S}^{*}, *, \lambda\right)$, where “*” denotes the step sequence concatenation operator (usually omitted), is a monoid of step sequences.

Definition 5 ([12]). Let $\theta=(E$, sim, ser $)$ be a comtrace alphabet and let $\equiv_{\text {ser }}$, called comtrace congruence, be the EQ-congruence defined by the set of equations:

$$
E Q=\{A=B C \mid A=B \cup C \in \mathbb{S} \wedge B \times C \subseteq \operatorname{ser}\} .
$$

Then the equational monoid $\left(\mathbb{S}^{*} / \equiv_{\text {ser }}, \circledast,[\lambda]\right)$ is called a monoid of comtraces over $\theta$.

We will omit the subscript ser from comtrace congruence if it causes no ambiguity.

Example 2. Let $E=\{a, b, c\}, \operatorname{sim}=\{(a, b),(b, a),(a, c),(c, a)\}$ and $\operatorname{ser}=\{(a, b),(b, a)$, $(a, c)\}$. Then we have $\mathbb{S}=\{\{a\},\{b\},\{c\},\{b, c\}\}$. A step sequence $s=\{a, b\}\{c\}\{c\}$ generates $[s]=\{\{a, b\}\{c\}\{c\},\{a\}\{b\}\{c\}\{c\},\{b\}\{a\}\{c\}\{c\},\{b\}\{a, c\}\{c\}\}$ as its comtrace. Note that $\{a\}\{c\}\{b\}\{a\} \notin[s]$.

Let $u=A_{1} \ldots A_{k}$ be a step sequence. By $\bar{u}=\bar{A}_{1} \ldots \bar{A}_{k}$ be the event enumerated representation of $u$. We will skip a lengthy but intuitively obvious formal definition (cf. [12]), but for instance, from Example 2, $\bar{s}=\left\{a^{(1)}, b^{(1)}\right\}\left\{c^{(1)}\right\}\left\{c^{(2)}\right\}$. Let $\Sigma_{u}=\bigcup_{i=1}^{k} \bar{A}_{i}$ denote the set of all enumerated events occurring in $u$, for example, $\Sigma_{s}=\left\{a^{(1)}, b^{(1)}, c^{(1)}, c^{(2)}\right\}$. For each $\alpha \in \Sigma_{u}$, let $\operatorname{pos}_{u}(\alpha)$ denote the consecutive number of a step where $\alpha$ belongs, i.e. if $\alpha \in \bar{A}_{j}$ then $\operatorname{pos}_{u}(\alpha)=j$. For our example, $\operatorname{pos}_{s}\left(c^{(2)}\right)=3, \operatorname{pos}_{s}\left(b^{(1)}\right)=1$, etc. For each enumerated even $\alpha=e^{(i)}$, let $l(\alpha)$ denote the label of $\alpha$, i.e. $l(\alpha)=$ $l\left(e^{(i)}\right)=e$. One can easily show that $u \equiv v \Longrightarrow \Sigma_{u}=\Sigma_{v}$, so we can define $\Sigma_{[u]}=\Sigma_{u}$.

Given a step sequence $u$, we define the stratified order $\triangleleft_{u} \subseteq \Sigma_{u} \times \Sigma_{u}$ induced by $u$ by: $\alpha \triangleleft_{u} \beta \Longleftrightarrow \operatorname{pos}_{u}(\alpha)<$ $\operatorname{pos}_{u}(\beta)$. Then it can be easily checked that the stratified orders induced by the step sequences of the comtrace $[s]$ from Example 2 are exactly the stratified extensions of the so-structure $S_{[s]}=\left(\Sigma_{[s]}, \prec_{[s]}, \sqsubset_{[s]}\right)$ on the right. The dotted edge denotes $\sqsubset_{[s]}$, while the solid edges denote

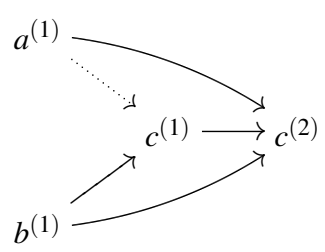
both $\prec_{[s]}$ and $\sqsubset_{[s]}$.

Analogous to Remark 1 for traces, given a comtrace alphabet $(E, \operatorname{sim}$, ser $)$ and a step sequence $u$, we do not need to analyse any other elements of $[u]$ except $u$ itself to construct the so-structure $S_{[u]}$, which the comtrace $[u]$ represents. We will now show how the $\diamond$-closure operator helps us to build the desired construction. 
Definition 6 ([12]). Let $u \in \mathbb{S}^{*}$. We define the relations $\prec_{u}, \sqsubset_{u} \subseteq \Sigma_{[u]} \times \Sigma_{[u]}$ as:

1. $\alpha \prec_{u} \beta \stackrel{d f}{\Longleftrightarrow} \alpha \triangleleft_{u} \beta \wedge(l(\alpha), l(\beta)) \notin$ ser,

2. $\alpha \sqsubset_{u} \beta \stackrel{d f}{\Longleftrightarrow} \alpha \triangleleft_{u} \beta \wedge(l(\beta), l(\alpha)) \notin$ ser.

Definition 6 describes two basic "local" invariants of the elements of $\Sigma_{\mathbf{u}}$. The relation $\prec_{u}$ captures the situation when $\alpha$ always precedes $\beta$, and the relation $\sqsubset_{u}$ captures the situation when $\alpha$ never follows $\beta$. However, since $\prec_{u}$ and $\sqsubset_{u}$ are "locally" invariant, the relation structure $\left(\Sigma_{[u]}, \prec_{u},\left\llcorner_{u}\right)\right.$ might not contain "global" invariants that can be inferred from (S3) and (S4) of Definition 2. For instance, the step sequence $s$ from Example 2 generates the following relations $\prec_{s}=\left\{\left(b^{(1)}, c^{(1)}\right),\left(b^{(1)}, c^{(2)}\right),\left(c^{(1)}, c^{(2)}\right)\right\}$ and $\sqsubset_{s}=\prec_{s} \cup\left\{\left(a^{(1)}, c^{(1)}\right),\left(a^{(1)}, c^{(2)}\right)\right\}$, where the edge $\left(a^{(1)}, c^{(2)}\right)$ from $\prec_{[s]}$ is absent from $\prec_{s}$. To make sure all invariants are included, we need $\diamond$-closure.

Definition 7. Given a step sequence $u \in \mathbb{S}^{*}$ and its respective comtrace $[u] \in \mathbb{S}^{*} / \equiv$. We define the relational structures $S_{[u]}$ as: $\quad S_{[u]} \stackrel{d f}{=}\left(\Sigma_{[u]}, \prec_{u}, \sqsubset_{u}\right)^{\diamond}$.

The relational structure $S_{[u]}$ is the so-structure defined by the comtrace $[u]$. The following theorem justifies the names and summarises the following nontrivial results concerning the so-structures generated by comtraces. The proofs of these results heavily use the properties of $\diamond$-closure from Theorem 4 .

Theorem $5([12,13])$. For all $u, v \in \mathbb{S}^{*}$, we have

1. $S_{[u]}$ is a so-structure and $S_{[u]}=\left(\Sigma_{[u]}, \bigcap_{x \in[u]} \triangleleft_{x}, \bigcap_{x \in[u]} \triangleleft_{x}\right)$,

2. $u \equiv v \Longleftrightarrow S_{[u]}=S_{[v]}$,

3. $\operatorname{ext}\left(S_{[u]}\right)=\left\{\triangleleft_{s} \mid s \in[u]\right\}$.

Note that a generalisation of Theorem 5 to generalised stratified order structures (gso-structures) [9], an extension of so-structures which can additionally model the "non-simultaneously" relationship, was recently shown in [14,20]. A sequence representation of gso-structures called generalised comtraces were proposed and shown to represent precisely finite gso-structures. The intuition of the approach in [20] is similar to what we discussed here and the $\diamond$-closure operator was applied extensively.

\section{Interval Order Structures and $\diamond$-closure}

This section contains the major contribution of this paper. We start with a short presentation of some properties on io-structures, then we define -closure, the main concept of this paper, and prove the equivalence of Theorem 4 . Because io-structures are more complex than so-structures, the proofs are more involved than that of Theorem 4.

Definition 8 ([11]). An interval order structure (io-structure) is a relational structure $S=(X, \prec, \sqsubset)$, such that for all $a, b, c, d \in X$, the following hold:

$$
\begin{aligned}
& \text { I1: } a \not \subset a \\
& \text { I4: } a \prec b \sqsubset c \vee a \sqsubset b \prec c \Longrightarrow a \sqsubset c \\
& \text { I2: } a \prec b \Longrightarrow a \sqsubset b \\
& \text { I5: } a \prec b \sqsubset c \prec d \Longrightarrow a \prec d \\
& \text { I3: } a \prec b \prec c \Longrightarrow a \prec c \\
& \text { I6: } a \sqsubset b \prec c \sqsubset d \Longrightarrow a \sqsubset d \vee a=d
\end{aligned}
$$


Here the causality relation $\prec$ also represents the "earlier than" relationship, and the weak causality relation $\sqsubset$ represents the "not later than" relationship but under the assumption that the system runs are interval orders.

\section{Proposition 2 ([11]).}

1. $\prec$ is a partial order such that $a \prec b \Rightarrow b \not \subset a$ and $a \sqsubset b \Rightarrow b \nprec a$.

2. If $<$ is an interval order on $X$, then $\left(X,<,<^{-}\right)$is an io-structure.

Interval order structures were independently introduced in [19] and [10]. Some of their properties have been presented in [13], yet their theory is not as well-developed and much less often applied than that of so-structures [9]. The lack of an operator analogous to the $\diamond$-closure prevented us from building a working relationship between iostructures and sequence models of concurrency such as Mazurkiewicz traces and comtraces.

Theorem 6 ([13]). Every so-structure is an io-structure.

Since every so-structure is an io-structure, many properties of so-structures also hold for io-structures. Furthermore, we also have an analogue of Theorem 3 for interval orders and io-structures.

An interval order $<$ on $X$ is an interval extension of an io-structure $S=(X, \prec, \sqsubset)$ if $\prec \subseteq<$ and $\sqsubset \subseteq<\urcorner$. The set of all interval extensions of $S$ will be denoted by $\operatorname{Interv}(S)$.

Theorem 7 ([13]). For each io-structure $S=(X, \prec, \sqsubset)$, we have

$$
\left.S=\left(X, \bigcap_{<\in \operatorname{lnterv}(S)}<, \bigcap_{<\in \operatorname{Interv}(S)}<\right\urcorner\right) .
$$

The above theorem is a generalisation of Szpilrajn's Theorem to io-structures. It is interpreted as the proof of the claim that io-structures uniquely represent sets of equivalent system runs, provided that the system's operational semantics can be fully described in terms of interval orders (see $[9,13]$ for details). An example of a simple interval order structure which illustrates the main ideas behind this concept is shown in Figure 2.

Before defining the concept of -closure and proving its properties, we need to introduce some auxiliary notions and prove some preliminary results.

Definition 9. Let $R_{1}, R_{2} \subseteq X \times X$ be two relations and let $\left\langle S_{1}, \ldots, S_{k}\right\rangle$ be a sequence of relations such that $S_{i} \in\left\{R_{1}, R_{2}\right\}, i=1, \ldots, k$.

1. A sequence $\left\langle S_{1}, \ldots, S_{k}\right\rangle$ has $\uplus$-property w.r.t. $\left(R_{1}, R_{2}\right)$, if for all $i, 1 \leq i<k$, we have $\neg\left(S_{i}=S_{i+1}=R_{2}\right)$, i.e. there are no two consecutive $R_{2}$ 's.

2. A sequence $\left\langle S_{1}, \ldots, S_{k}\right\rangle$ has $\oplus$-property w.r.t. $\left(R_{1}, R_{2}\right)$, if $k \geq 1, S_{1}=S_{k}=R_{1}$ and the sequence $\left\langle S_{2}, \ldots, S_{k-1}\right\rangle$ has $\uplus$-property w.r.t. $\left(R_{1}, R_{2}\right)$;

3. $R_{1} \uplus R_{2}=\bigcup_{k>0}\left\{S_{1} \circ \ldots \circ S_{k} \mid\left\langle S_{1}, \ldots, S_{k}\right\rangle\right.$ has $\uplus$-property w.r.t. $\left.\left(R_{1}, R_{2}\right)\right\}$.

4. $R_{1} \oplus R_{2}=\bigcup_{k \geq 1}\left\{S_{1} \circ \ldots \circ S_{k} \mid\left\langle S_{1}, \ldots, S_{k}\right\rangle\right.$ has $\oplus$-property w.r.t. $\left.\left(R_{1}, R_{2}\right)\right\}$. 

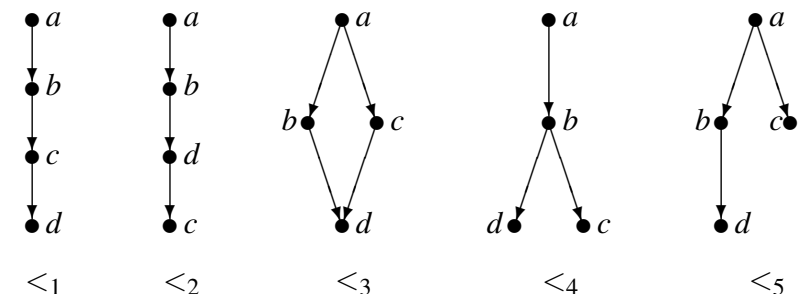

$<_{4}$
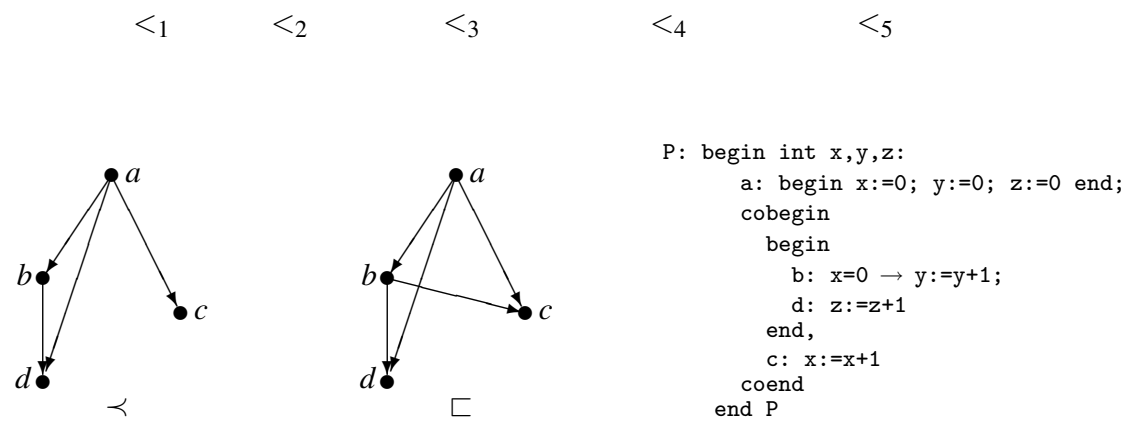

Fig. 2. An example of a simple interval order structure $S=(X, \prec, \sqsubset)$, with $X=\{a, b, c, d\}$ and its set of all interval extensions $\operatorname{Interv}(S)=\left\{<_{1},<_{2},<_{3},<_{4},<_{5}\right\}$. The orders $<_{1}$ and $<_{2}$ are total, $<_{3}$ and $<_{4}$ are stratified and $<_{5}$ is interval but not stratified. The elements of $\operatorname{Interv}(S)$ are all equivalent runs (executions) of the program $P$ involving the actions $a, b, c$ and $d$, so the interval order structure uniquely defines a concurrent behaviour (history) of $P$ (see [9] for details). The elements of Interv $(S)$ are represented as Hasse diagrams, while $\prec$ and $\sqsubset$ are represented as graphs of their entire relations. In this case $\prec$ equals $<_{5}$, as there are not so many partial orders over the four elements set, but the interpretations of $<_{5}$ and $\prec$ are different. The incomparability in $<_{5}$ is interpreted as simultaneity while in $\prec$ as having no casual relationship.

For example the sequence $\left\langle R_{1}, R_{2}, R_{2}, R_{1}\right\rangle$ has neither $\uplus$ - nor $\oplus$-property, the empty sequence \langle\rangle and the sequence $\left\langle R_{1}, R_{1}, R_{2}, R_{1}, R_{2}\right\rangle$ has $\uplus$-property but not $\oplus$-property, and $\left\langle R_{1}, R_{1}, R_{2}, R_{1}, R_{2}, R_{1}\right\rangle$ has $\oplus$-property. We will omit the suffix "w.r.t. $\left(R_{1}, R_{2}\right)$ " if the relations $R_{1}$ and $R_{2}$ are clear from the context. The relations $R_{1} \oplus R_{2}$ and $R_{1} \uplus R_{2}$ can easily be defined by appropriate regular expressions built from $R_{1}$ and $R_{2}$.

Proposition 3. Let $R_{1}, R_{2} \subseteq X \times X$ be two relations. Then

1. $R_{1} \oplus R_{2}=\left(R_{1}^{+} \circ R_{2}\right)^{*} \circ R_{1}^{+}$,

2. $R_{1} \uplus R_{2}=\left(R_{2} \cup i d_{X}\right) \circ\left(R_{1} \cup R_{1} \circ R_{2}\right)^{*}$,

3. $R_{1} \oplus R_{2} \subseteq R_{1} \uplus R_{2}$,

4. $\left(R_{1} \oplus R_{2}\right) \uplus\left(R_{1} \uplus R_{2}\right) \subseteq\left(R_{1} \uplus R_{2}\right)$,

5. $\left(R_{1} \oplus R_{2}\right) \oplus\left(R_{1} \uplus R_{2}\right) \subseteq\left(R_{1} \oplus R_{2}\right)$.

Proof. Follows immediately from Definition 9.

We can now define the main concept of this paper, the concept of $\$$-closure.

Definition 10. For every relational structure $S=\left(X, R_{1}, R_{2}\right)$ we define $S^{\bullet}$, the -closure of $S$, as:

$$
S \stackrel{d f}{=}\left(X, \prec \gtrless_{R_{1}, R_{2}}, \sqsubset \bullet_{R_{1}, R_{2}}\right)=\left(X, R_{1} \oplus R_{2},\left(R_{1} \uplus R_{2}\right) \backslash i d_{X}\right) .
$$


The $\diamond$-closure is an extension of $\diamond$-closure of so-structures and transitive closure of relations to io-structures. We will start by proving equivalences of Theorem $4(1,2)$.

Proposition 4. 1. If $R_{2}$ is irreflexive, then $S \subseteq S^{\star} . \quad$ 2. $\left(S^{\star}\right)^{\star}=S^{\star}$.

Proof. 1. By the definition $R_{1} \subseteq R_{1} \oplus R_{2}=\prec_{R_{1}, R_{2}}$ and $R_{2} \subseteq R_{1} \uplus R_{2}$. Hence, if $R_{2}$ is irreflexive, $R_{2} \backslash i d_{X} \subseteq\left(R_{1} \uplus R_{2}\right) \backslash i d_{X}=\sqsubset R_{1}, R_{2}$.

2. () Since $\sqsubset R_{1}, R_{2}$ is irreflexive, by (1) we have $S \subseteq\left(S^{\bullet}\right.$.

$(\subseteq)$ We need to show that $\prec \prec$ means $\left(R_{1} \oplus R_{2}\right) \oplus\left(R_{1} \uplus R_{2}\right) \subseteq R_{1} \oplus R_{2}$, and $\left(R_{1} \oplus R_{2}\right) \uplus\left(R_{1} \uplus R_{2}\right) \subseteq R_{1} \uplus R_{2}$. But this follows from Proposition 3(4,5).

Proposition 4(2) states that $\diamond$-closure is idempotent, and justifies the name closure (cf. [24]).

Note that the exact replica of Theorem 4(3) is false. Consider an example, where $X=\{a, b\}, R_{1}=\{(a, b)\}$ and $R_{2}=\{(b, a)\}$. Thus, $\prec_{R_{1}, R_{2}}=\{(a, b)\}$ and $\sqsubset R_{1}, R_{2}=$ $\{(a, b),(b, a)\}$, so $\prec_{R_{1}, R_{2}}$ is irreflexive, but $\left(X, \prec_{R_{1}, R_{2}}, \sqsubset R_{1}, R_{2}\right)$ is not an io-structure since $a \prec_{R_{1}, R_{2}} b \sqsubset \gtrless_{R_{1}, R_{2}} a \Longrightarrow a \sqsubset a$, which contradicts (I1) from Definition 8. To find the necessary and sufficient condition for the $\downarrow$-closure of a relational structure to be an io-structure, we need a new concept.

Definition 11. A relational structure $S=\left(X, R_{1}, R_{2}\right)$ is i-directed if

1. $R_{1} \oplus R_{2}$ is irreflexive, and

2. $\forall a, b \in X .(a, b) \in R_{2} \Longrightarrow(b, a) \notin R_{1} \oplus R_{2}$.

Proposition 5. $S^{\Downarrow}$ is an io-structure if and only if $S=\left(X, R_{1}, R_{2}\right)$ is i-directed.

Proof. $\left(\Rightarrow\right.$ ) If $S \downarrow$ is an io-structure then by (I1) and (I2), $\prec R_{1}, R_{2}=R_{1} \oplus R_{2}$ is irreflexive. Suppose $(a, b) \in R_{2}$ and $(b, a) \in R_{1} \oplus R_{2}$. Since $R_{2} \subseteq \sqsubset R_{1}, R_{2}$, we have $a \prec \gtrless_{R_{1}, R_{2}} b$ and $b \sqsubset R_{1}, R_{2} a$, which contradicts Proposition 2(1).

$(\Leftarrow)$ We need to show that the conditions of Definition 8 are satisfied.

(I1) Clearly $\left(R_{1} \uplus R_{2}\right) \backslash i d_{X}$ is irreflexive.

(I2) From Corollary 3(3) we have $\prec{ }_{R_{1}, R_{2}} \subseteq R_{1} \uplus R_{2}$. Since $\prec{ }_{R_{1}, R_{2}}$ is irreflexive, $\prec{ }_{R_{1}, R_{2}} \subseteq$ $\left(R_{1} \uplus R_{2}\right) \backslash i d_{X}=\sqsubset R_{1}, R_{2}$.

(I3) Let $a \prec_{R_{1}, R_{2}} b$ and let $b \prec R_{R_{1}, R_{2}} c$. This means $a S_{1} \circ \ldots \circ S_{k} b Q_{1} \circ \ldots \circ Q_{r} c$, where $\left\langle S_{1}, \ldots, S_{k}\right\rangle$ and $\left\langle Q_{1}, \ldots, Q_{r}\right\rangle$ both have $\oplus$-property. Hence $\left\langle S_{1}, \ldots, S_{k}, Q_{1}, \ldots, Q_{r}\right\rangle$ also has $\oplus$-property. Thus, $a \prec_{R_{1}, R_{2}} c$.

(I4) Let $a \prec_{R_{1}, R_{2}} b$ and let $b \sqsubset_{R_{1}, R_{2}} c$. This means $a S_{1} \circ \ldots \circ S_{k} b Q_{1} \circ \ldots \circ Q_{r} c$, where $\left\langle S_{1}, \ldots, S_{k}\right\rangle$ satisfies $\oplus$-property, and $\left\langle Q_{1}, \ldots, Q_{r}\right\rangle$ satisfies $\uplus$-property. Hence the sequence $\left\langle S_{1}, \ldots, S_{k}, Q_{1}, \ldots, Q_{r}\right\rangle$ has $\uplus$-property and thus $(a, c) \in R_{1} \uplus R_{2}$. Suppose $a=c$. Since $a \prec_{R_{1}, R_{2}} b$ and $b \sqsubset R_{1}, R_{2} c$, this means $a R_{1} \circ S_{1} \circ \ldots \circ S_{k} \circ R_{1} b$, and $b Q_{1} \circ Q_{2} \circ \ldots \circ Q_{s-1} \circ Q_{s} a$, where $S_{i}, Q_{i} \in\left\{R_{1}, R_{2}\right\}$. Either $Q_{1}$ or $Q_{s}$ are equal to $R_{2}$, 
otherwise $b \prec_{R_{1}, R_{2}} a$, contradicting that $\prec_{R_{1}, R_{2}}$ is irreflexive. Suppose $Q_{1}=R_{2}$. This means $Q_{2}=R_{1}$. Thus there is some $b_{1}$ such that $b R_{2} b_{1} R_{1} \circ Q_{3} \circ \ldots \circ Q_{s} \circ R_{1} \circ S_{1} \circ$ $\ldots \circ S_{k} \circ R_{1} b$, which means $\left(b_{1}, b\right) \in R_{1} \oplus R_{2}$, contradicting Definition 11(2). Hence $Q_{1}=R_{1}$ and $Q_{s}=R_{2}$, i.e. $Q_{s-1}=R_{1}$. Thus, there is some $b_{s}$ such that $b_{s} R_{2} a$ and $a R_{1} \circ S_{1} \circ \ldots \circ S_{k} \circ R_{1} \circ Q_{1} \circ \ldots \circ R_{1} b_{s}$, which means $\left(a, b_{s}\right) \in R_{1} \oplus R_{2}$, contradicting Definition 11(2). Therefore $a \neq c$, i.e. $(a, c) \in\left(R_{1} \uplus R_{2}\right) \backslash i d_{X}=\sqsubset R_{1}, R_{2}$.

For the case when $a \sqsubset R_{1}, R_{2} b \prec_{R_{1}, R_{2}} c$, we proceed almost identically.

(I5) Let $a \prec_{R_{1}, R_{2}} b \sqsubset_{R_{1}, R_{2}} c \prec_{R_{1}, R_{2}} d$. Thus, there are sequences $\left\langle S_{1}, \ldots, S_{k}\right\rangle,\left\langle P_{1}, \ldots, P_{s}\right\rangle$ and $\left\langle Q_{1}, \ldots, Q_{r}\right\rangle$, such that $a S_{1} \circ \ldots \circ S_{k} b P_{1} \circ \ldots \circ P_{s} c Q_{1} \circ \ldots \circ Q_{r} d$, where $\left\langle S_{1}, \ldots, S_{k}\right\rangle$ and $\left\langle Q_{1}, \ldots, Q_{r}\right\rangle$ have $\oplus$-property and $\left\langle P_{1}, \ldots, P_{s}\right\rangle$ has $\uplus$-property. It follows that $\left\langle S_{1}, \ldots, S_{k}, Q_{1}, \ldots, Q_{r}, P_{1}, \ldots, P_{s}\right\rangle$ has $\oplus$-property and thus $a \prec{ }_{R_{1}, R_{2}} d$.

(I6) Let $a \sqsubset R_{1, R_{2}} b \prec_{R_{1}, R_{2}} c \sqsubset R_{R_{1}, R_{2}} d$. Thus, there are sequences $\left\langle S_{1}, \ldots, S_{k}\right\rangle,\left\langle P_{1}, \ldots, P_{s}\right\rangle$ and $\left\langle Q_{1}, \ldots, Q_{r}\right\rangle$, such that $a S_{1} \circ \ldots \circ S_{k} b P_{1} \circ \ldots \circ P_{s} c Q_{1} \circ \ldots \circ Q_{r} d$, where $\left\langle S_{1}, \ldots, S_{k}\right\rangle$ and $\left\langle Q_{1}, \ldots, Q_{r}\right\rangle$ have $\uplus$-property and $\left\langle P_{1}, \ldots, P_{s}\right\rangle$ has $\oplus$-property. It follows that $\left\langle S_{1}, \ldots, S_{k}, Q_{1}, \ldots, Q_{r}, P_{1}, \ldots, P_{S}\right\rangle$ has $\uplus$-property. So $a \sqsubset R_{1}, R_{2} b$ or $a=d$.

The fact that the above result is slightly weaker than Theorem 4(3) does not seem to matter much as in virtually all applications of $\diamond$-closure in [12] and [17], the relations $R_{1}$ and $R_{2}$ satisfy the equivalence of the conditions of Definition 11 for so-structures. The below result appears to be quite useful for various potential applications of $\downarrow$-closure.

Proposition 6. Let $S=\left(X, R_{1}, R_{2}\right)$ be a relational structure and let $<\subseteq X \times X$ be an interval order such that $R_{1} \subseteq<$ and $R_{2} \subseteq<$. Then $S$ is i-directed.

Proof. By Proposition 2(2), $(X,<,<\frown)$ is an io-structure, so it satisfies I1-I6. We have $R_{1}^{+} \subseteq<^{+}=<$, so $R_{1} \oplus R_{2}=\left(R_{1}^{+} \circ R_{2}\right)^{*} \circ R_{1}^{+} \subseteq\left(<\circ R_{2}\right)^{*} \circ<=\bigcup_{i=0}^{\infty}\left(\left(<\circ R_{2}\right)^{i} \circ<\right)$. For each $i$, we have $\left(<\circ R_{2}\right)^{i} \circ<\subseteq(<\circ<\frown)^{i} \circ<$ and then by applying (I5) $i$ times, we have $(<\circ<\frown)^{i} \circ<\subseteq<$. Hence $R_{1} \oplus R_{2} \subseteq<$. i.e. $R_{1} \oplus R_{2}$ is irreflexive. If $(a, b) \in R_{2}$ then $a<\succ b$, i.e. $\neg(b<a)$ and also $(a, b) \notin R_{1} \oplus R_{2}$ as $R_{1} \oplus R_{2} \subseteq<$.

Both $\diamond$ - and $\diamond$-closures are often used for the cases like the one in Definition 7 , so we can then use the above results to simplifies the proofs.

We now prove an analogue of Theorem 4(4), which states that io-structures are fixed points of $\diamond$-closure.

Proposition 7. If $S=(X, \prec, \sqsubset)$ is an io-structure then $S=S$.

Proof. ( $\subseteq$ ) Since $S$ is an io-structure, $\sqsubset$ is irreflexive. Thus, by Proposition 4(1), $S \subseteq S^{\downarrow}$. () We will first show that $\prec \oplus \sqsubset \subseteq \prec$. Since $\prec \oplus \sqsubset=\left(\prec^{+} \circ \sqsubset\right)^{*} \circ \prec^{+}$, it suffices to show that for each $i \geq 1, j \geq 0, k \geq 1,\left(\prec^{i} \circ \sqsubset\right)^{j} \circ \prec^{k} \subseteq \prec$. From (I3) it follows $\prec^{i} \subseteq \prec$ and $\prec^{k} \subseteq \prec$, so $\left(\prec^{i} \circ \sqsubset\right)^{j} \circ \prec^{k} \subseteq(\prec \circ \sqsubset)^{j} \circ \prec$. By apply (I5) from right to left $i$ times, we have $(\prec \circ \sqsubset)^{j} \circ \prec \subseteq \prec$. Thus, $\prec \oplus \sqsubset \subseteq \prec$.

It remains to show $(\prec \uplus \sqsubset) \backslash i d_{X} \subseteq \sqsubset$. By Proposition 3(2), $\uplus \sqsubset=\left(\sqsubset \cup i d_{X}\right) \circ(\prec$ $\cup \prec \circ \sqsubset)^{*}$. It suffices to show that for all $i \geq 0,\left(\sqsubset \cup i d_{X}\right) \circ(\prec \cup \prec \circ \sqsubset)^{i} \subseteq \sqsubset \cup i d_{X}$. The case when $i=0$ is trivial. For $i>0$, by the induction hypothesis, we have $\left(\sqsubset \cup i d_{X}\right) \circ(\prec$ $\cup \prec \circ \sqsubset)^{i-1} \subseteq \sqsubset \cup i d_{X}$. It suffices to show $\left(\sqsubset \cup i d_{X}\right) \circ(\prec \cup \prec \circ \sqsubset) \subseteq \sqsubset \cup i d_{X}$. But this holds since, by (I4) and (I6), (( $\left.\left.\sqcup \cup i d_{X}\right) \circ \prec\right) \cup\left(\left(\sqsubset \cup i d_{X}\right) \circ \prec \circ \sqsubset\right) \subseteq \sqsubset \cup i d_{X}$. 
Directly from Proposition 7 we obtain the below result which will be used in the proof of the analogue of Theorem 4(5).

Corollary 1. Every io-structure is i-directed.

Proposition 8. Let $S=(X, \prec, \sqsubset)$ be an io-structure and let $S_{0} \subseteq S$. Then $S_{0}^{\bullet} \subseteq S$ and $S_{0}^{\bullet}$ is an io-structure.

Proof. From Proposition 7 it immediately follows $S_{0}^{\bullet} \subseteq S^{\Downarrow}=S$.

Due to Proposition 5 it suffices to show that $S_{0}$ is i-directed. Let $S_{0}=\left(X, R_{1}, R_{2}\right)$. We have $R_{1} \oplus R_{2} \subseteq \prec \oplus \sqsubset={ }^{\text {(Proposition 7) }} \prec$. Since $\prec$ is irreflexive, $R_{1} \oplus R_{2}$ is irreflexive as well. Let $(a, b) \in R_{2}$. Since $R_{2} \subseteq \sqsubset$, we have $a \sqsubset b$ which by Corollary 1 , implies $(b, a) \notin \prec \oplus \sqsubset$. Since $R_{1} \oplus R_{2} \subseteq \prec \oplus \sqsubset,(b, a) \notin R_{1} \oplus R_{2}$. Therefore $S_{0}$ is i-directed.

We can also show that $\diamond$-closure is indeed a generalisation of $\diamond$-closure.

Proposition 9. If $S$ is so-structure then $S=S^{\diamond}=S^{\bullet}$.

Proof. A consequence of Theorem 4(4), Theorem 6 and Proposition 7.

\section{Final Comments}

A concept of -closure has been defined for io-structures. It is an equivalence of $\diamond$ closure of so-structures ([12]) and classical transitive closure of relations. It has also been proven that, in principle, $\checkmark$-closure has the same properties as $\diamond$-closure and transitive closure. Because the definition of -closure was more elaborate, the proofs were substantially more complex than their counterparts for $\diamond$-closure. Nevertheless, only one property of $\checkmark$-closure is slightly weaker than its $\diamond$-closure counterpart.

The counterpart of comtraces for io-structures has not been fully developed yet, but its foundation has been established. Fishburn's Theorem (Theorem 2) states that each interval order can be represented by an appropriate total order of the interval beginnings and ends. The below fundamental theorem states that each io-structure can be represented by an appropriate partial (not necessarily interval) order of the beginnings and ends.

Theorem 8 (Abraham, Ben-David, Magodor [1]).

A relational structure $S=(X, \prec, \sqsubset)$ is an io-structure iff there exists a partial order $\triangleleft$ on some $Y$ and two mappings $B, E: X \rightarrow Y$ such that $B(X) \cap E(X)=\emptyset$ and for each

$$
\text { 1. } B(x) \triangleleft E(x), \quad \text { 2. } x \prec y \Longleftrightarrow E(x) \triangleleft B(y), \quad \text { 3. } x \sqsubset y \Longleftrightarrow B(x) \triangleleft E(y) \text {. }
$$

Szpilrajn's Theorem (Theorem 1) allows us to represent each partial order by its total extensions. The combination of these three theorems and Theorem 7 makes it possible to construct "interval traces", a version of Mazurkiewicz traces over an appropriate monoid of sequences of beginnings and ends, and then use "interval traces" to represent io-structures via Theorem 8. This topic is beyond the scope of this paper; however, the properties of -closure are essential tools in this process. 
Acknowledgement. The authors thank all three anonymous referees whose comments have helped us improve the final version of this paper significantly.

\section{References}

1. Abraham, U., Ben-David, S., Magodor, M.: On global-time and inter-process communication. In: Semantics for Concurrency, Workshops in Computing, pp. 311-323. Springer (1990)

2. Begstra, J.A. et al (eds.): The Handbook of Process Algebras. Elsevier Science (2000)

3. Burris, S., Sankappanavar, H.P.: A Course in Universal Algebra. Springer, New York (1981)

4. Best, E., Koutny, M.: Petri net semantics of priority systems. Theoretical Computer Science 94, pp. 141-158 (1992)

5. Diekert, V., Rozenberg, G. (eds.): The Book of Traces. World Scientific (1995)

6. Fishburn, P.C.: Intransitive indifference with unequal indifference intervals. Journal of Mathematical Psychology, vol. 7, pp. 144-149 (1970)

7. Fishburn, P. C.: Interval Orders and Interval Graphs. John Wiley, New York (1985)

8. Gaifman, H., Pratt, V.: Partial Order Models of Concurrency and the Computation of Function. In: Proc. LICS 1987, pp. 72-85 (1987)

9. Janicki, R.: Relational Structures Model of Concurrency. Acta Informatica 45(4), pp. 279320 (2008)

10. Janicki, R., Koutny, M.: Invariants and Paradigms of Concurrency Theory. In: Proc. PARLE 1991. LNCS, vol. 506, pp. 59-74. Springer (1991)

11. Janicki, R., Koutny, M.: Structure of Concurrency. Theoretical Computer Science 112(1), pp. 5-52 (1993)

12. Janicki, R., Koutny, M.: Semantics of Inhibitor Nets. Information and Computation 123(1), pp. 1-16 (1995)

13. Janicki, R., Koutny, M.: Fundamentals of Modelling Concurrency Using Discrete Relational Structures. Acta Informatica 34, pp. 367-388 (1997)

14. Janicki, R., Lê, D.T.M.: Modelling Concurrency with Quotient Monoids. In: Proc. ATPN 2008. LNCS, vol. 5062, pp. 251-269. Springer (2008)

15. Jensen, K.: Coloured Petri Nets. Vol. 1,2,3. Springer (1997)

16. Juhás, G., Lorenz, R., Mauser, S.: Synchronous + Concurrent + Sequential $=$ Earlier than + Not later than. In: Proc. ACSD 2006, pp. 261-272. IEEE (2006)

17. Kleijn, H.C.M., Koutny, M.: Process Semantics of General Inhibitor Nets. Information and Computation 190, pp. 18-69 (2004)

18. Kleijn, J., Koutny, M.: Formal Languages and Concurrent Behaviour. Studies in Computational Intelligence 113, pp. 125-182 (2008)

19. Lamport, L.,: The mutual exclusion problem: Part I - a theory of interprocess communication; Part II - statements and solutions. Journal of ACM 33(2), pp. 313-326 (1986)

20. Lê, D.T.M.: Studies in Comtrace Monoids. Master Thesis, Department of Computing and Software, McMaster University (2008)

21. Mazurkiewicz, A.: Concurrent Program Schemes and Their Interpretation. TR DAIMI PB78, Comp. Science Depart., Aarhus University (1977)

22. Milner, R.: Operational and Algebraic Semantics of Concurrent Processes. In: van Leuween, J. (ed.): Handbook of Theoretical Computer Science. Vol. 2, pp. 1201-1242. Elsevier (1993)

23. Reisig, W.: Elements of Distributed Algorithms. Springer (1998)

24. Rosen, K. H.: Discrete Mathematics and Its Applications. McGraw-Hill (1999)

25. Szpilrajn, E., Sur l'extension de l'ordre partiel. Fundam. Mathematicae 16, pp. 386-389 (1930)

26. Wiener, N.: A contribution to the Theory of Relative Position. In: Proc. Camb. Philos. Soc. 17, pp. 441-449 (1914) 